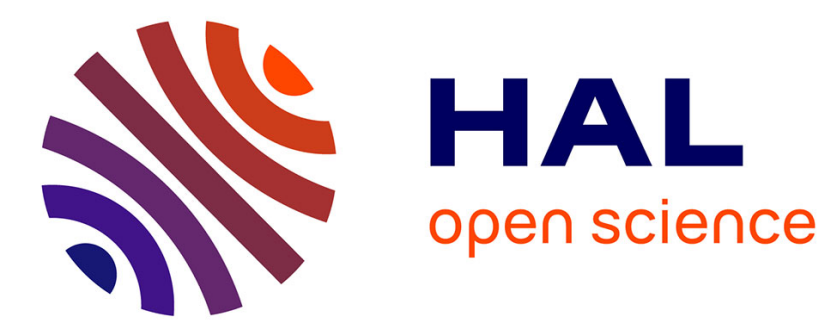

\title{
Chronique de droit pénal et de procédure pénale
} Évelyne Bonis, Virginie Peltier

\section{To cite this version:}

Évelyne Bonis, Virginie Peltier. Chronique de droit pénal et de procédure pénale. Titre VII - Les Cahiers du Conseil constitutionnel, 2020, 5, pp.112-125. 10.3917/tvii.005.0112 . hal-03472516

\section{HAL Id: hal-03472516 https://hal.science/hal-03472516}

Submitted on 14 Feb 2022

HAL is a multi-disciplinary open access archive for the deposit and dissemination of scientific research documents, whether they are published or not. The documents may come from teaching and research institutions in France or abroad, or from public or private research centers.
L'archive ouverte pluridisciplinaire HAL, est destinée au dépôt et à la diffusion de documents scientifiques de niveau recherche, publiés ou non, émanant des établissements d'enseignement et de recherche français ou étrangers, des laboratoires publics ou privés. 


\section{Chronique de droit pénal et de procédure pénale}

(janvier 2020 à juin 2020)

\section{Évelyne BONIS}

Professeur à l'Université de Bordeaux, Institut de sciences criminelles et de la justice

\section{Virginie PELTIER}

Professeur à l'Université de Bordeaux, Institut de sciences criminelles et de la justice

\section{Droit pénal général/droit pénal spécial}

Cons. const., déc. $n^{\circ}$ 2020-845 QPC du 19 juin 2020,

M. Théo S. (Recel d'apologie

du terrorisme). Article 421-2-5 du

Code pénal. Conformité. Réserve

d'interprétation.

P. Conte: Dr. pén. 2020, comm. à paraître, A. Gogorza : lexbase

\section{A85303NA, par V. PELTIER}

Cette décision fait suite à la question posée au Conseil par un détenu, condamné pour recel d'apologie d'acte de terrorisme en récidive à quatre ans d'emprisonnement, car trouvé en possession d'une clé USB, remise par sa compagne lors d'un parloir, contenant une soixantaine d'heures d'images de propagande de Daesh. Il soulève la question suivante qui met en réalité en cause, non pas la conformité à la Constitution d'une disposition légale, mais l'interprétation qui en est faite par la Cour de cassation : "Les dispositions combinées des articles 321-1 et 4212-5 du Code pénal, telles qu'interprétées par la jurisprudence de la Cour de cassation, en ce qu'elles incriminent, sous la qualification de recel d'apologie du terrorisme, la consultation de sites internet faisant l'apologie du terrorisme, ou la possession d'un support informatique ou numérique sur lequel serait téléchargé le produit d'une telle consultation, sont-elles contraires aux droits et libertés que la Constitution garantit et, plus précisément, au principe de la liberté 
d'opinion et de communication garanti par l'article 11 de la Déclaration des droits de l'homme et du citoyen de 1789, et méconnaissent-elles l'autorité de la chose jugée des décisions du Conseil constitutionnel?».

Comme souvent, le Conseil commence par examiner la recevabilité de la demande pour statuer au fond.

En premier lieu, il conclut à la recevabilité de la question prioritaire de constitutionnalité présentée en dépit de sa décision du 18 mai 2018 dans laquelle il avait déjà déclaré la portion de phrase déférée conforme à la Constitution (Cons. const., déc. $n^{\circ}$ 2018-706 QPC du 18 mai 2018: Comm. com. électr. 2009, étude 17, P. Auvret

C'est donc, en second lieu, la jurisprudence de la Cour de cassation qui était soumise à examen, sous couvert de celle du texte relatif à l'apologie, plus précisément un arrêt du 7 janvier dans lequel la Cour a indiqué qu' " entre dans les prévisions des articles 321-1 et 421-2-5 du Code pénal le fait de détenir, à la suite d'un téléchargement effectué en toute connaissance de cause, des fichiers caractérisant l'apologie d'actes de terrorisme », étant entendu malgré tout qu' " une condamnation de ce chef n'est compatible avec l'article 10 de la Convention européenne des droits de l'homme que si est caractérisée, en la personne du receleur, son adhésion à l'idéologie exprimée dans de tels fichiers " (Cass. crim., 7 janv. 2020, $n^{\circ}$ 19-80136 : Dr. pén. 2020, comm. 48, obs. P. Conte; JCP G 2020, 341, note G. Beaussonie ; F. Safi, comm., Lexbase Pénal, mars $2020 N^{\circ}$ Lexbase : N2501BYB; V. Malabat, Recel de biens provenant d'apologie d'actes de terrorisme, RPDP 2020, p. 121)

Le Conseil, tout en s'abstenant d'abroger le texte, ne serait-ce que partiellement, va cependant briser la jurisprudence de la Cour de cassation estimant que le délit de recel d'apologie d'acte terroriste porte à la liberté d'expression et de communication, garantie par l'article 11 de la Déclaration des droits de l'homme et du citoyen, une atteinte «qui n'est pas nécessaire, adaptée et proportionnée ». Son raisonnement s'effectue en deux temps.

Tout d'abord, à l'image du raisonnement tenu dans ses décisions relatives au délit de consultation de sites terroristes (Cons. const., déc. $n^{\circ}$ 2016-611 QPC du 10 fév. 2017,: Dr. pén. 2017, comm. 174, obs. P. Conte; JCP G 2017, 343, note A. Gogorza et B. de Lamy ; D. 2018. 1344, obs. E. Debaets et N. Jacquinot; AJ pénal 2017. 237, obs. J. Alix ; Dalloz IP/IT 2017. 289, obs. M. Quéméner ; RSC 2018. 75, obs. P. Beauvais ; Cons. const., déc. $n^{\circ}$ 2017-682 QPC du 15 déc. 2017, M. David P. : JCP G 2018, 109, note A. Gogorza et B. de Lamy ; Dr. pén. 2018, comm. 22, obs. P. Conte ; AJDA 2017. 2499 ; Conte ; D. 2018. 97, note Y. Mayaud; ibid. 1344, obs. E. Debaets et N. Jacquinot; RSC 2018. 75, obs. P. Beauvais ; O. Cahn, Délit de consultation de sites terroristes : ni fleurs, ni couronnes..., Lexbase Pénal, janvier 2018 ( $N^{\circ}$ Lexbase : N2232BXX)), le Conseil commence par rappeler que le droit pénal positif compte de nombreuses dispositions pour lutter contre le terrorisme, en plus de l'article 421-2-5 déféré, complétées par des dispositions du Code de la sécurité intérieure, susceptibles d'être appliquées au terme d'investigations de la part des autorités judicaires comme administratives, auxquelles ont été offerts par le législateur de larges moyens procéduraux. Ainsi, le Conseil conclut que, s'agissant de la condition de nécessité, il existe de nombreux moyens pour surveiller et sanctionner les personnes qui collectent des messages relatifs à une entreprise terroriste dès lors que ces agissements « s'accompagnent d'un comportement révélant une intention terroriste, avant même que ce projet soit entré dans sa phase d'exécution ».

C'est davantage au regard, ensuite, de l'adaptation et de la proportionnalité de l'atteinte portée par le délit de recel d'apologie que le bât va blesser tant sur le plan du fond que de celui de la répression. En effet, d'une part, le recel d'apologie ne suppose pas que son auteur ait l'intention de commettre un acte terroriste ou même 
de faire à son tour l'apologie des actes, puisque la Cour de cassation n'exige qu'une « adhésion » aux thèses apologétiques soutenues de sorte que, d'autre part, les peines encourues du fait de l'application des peines du recel peuvent être de cinq, sept ou même dix ans d'emprisonnement, alors même que l'agent n'a aucune intention terroriste ou aucune volonté de faire lui-même l'apologie d'actes terroristes. C'est la raison pour laquelle le Conseil conclut que, si l'article 421-2-5 du Code pénal ne contrevient pas à la Constitution, l'interprétation qui en est faite - lorsque l'application de ce texte est combinée avec celle de la qualification de recel - ne peut servir à réprimer la détention de documents contenant l'apologie d'actes de terrorisme, car elle porte une atteinte à la liberté d'expression qui n'est ni adaptée ni proportionnée. On en revient donc à la position (quasi) initiale du Conseil, puisque, dans sa première décision relative au délit de consultation de sites internet terroristes, il avait déploré que soit incriminé le fait de consulter des sites terroristes sans la preuve d'une intention de la part de l'agent de commettre un tel acte ou d'adhérer à l'idéologie véhiculée puis, dans la seconde, regretté l'absence d'intention, alors que le législateur avait rajouté à sa version de la qualification pénale la nécessité d'une adhésion de l'auteur aux thèses soutenues. Le critère de l'adhésion introduit par la Cour de cassation pour recréer, en définitive, le délit de consultation qui n'a jamais pu voir le jour, reste donc insuffisant. Or, restreindre la possibilité de sanction à la preuve de l'intention de commettre un acte de celui qui détient des images de propagande réduit à néant la pertinence d'une telle qualification.
Cons. const., déc. n ${ }^{\circ}$ 2020-799

DC du 26 mars 2020, Loi

organique d'urgence pour faire

face à l'épidémie de covid-19;

Cons. const., déc. n 2020-800 DC

du 11 mai 2020, Loi prorogeant

l'état d'urgence sanitaire et

complétant ses dispositions. JCP

Ent et aff, 9 juill. 2020, act. 476,

obs. J. Gallois, par V. PELTIER

Pour faire face à la pandémie liée à la propagation du covid 19, l'exécutif n'a eu d'autre choix que de proclamer un état d'urgence sanitaire, ce qui lui a permis par la suite de régir par une série d'ordonnances la vie économique, sanitaire et sociale de notre pays. La loi $n^{\circ} 2020-290$ du 23 mars 2020 d'urgence pour faire face à l'épidémie de covid-19 a ainsi été le socle des ordonnances du 25 mars, dont l'ordonnance $\mathrm{n}^{\circ}$ 2020-303 portant adaptation des règles de procédure pénale. Parallèlement, une loi organique d'urgence $n^{\circ}$ 2020-365 vit le jour le 30 mars 2020 pour faire face à l'épidémie de covid-19 et son article fut déféré au Conseil constitutionnel qui la déclara conforme à la Constitution. Cette disposition suspendait jusqu'au 30 juin 2020 le délai dans lequel le Conseil d'État ou la Cour de cassation doit normalement se prononcer sur le renvoi d'une question prioritaire de constitutionnalité au Conseil ainsi que le délai dans lequel celui-ci doit statuer en réponse. Dans la mesure où le texte ne remet en cause ni l'exercice du recours à la QPC, ni la possibilité qu'il soit statué sur une question posée, le Conseil valida l'article unique dans sa décision du 26 mars 2020.

La décision du 11 mai est plus fournie puisqu'elle se penche sur la loi n ${ }^{\circ} 2020-546$ du 11 mai 2020 prorogeant l'état d'urgence sanitaire et, surtout, complétant ses dispositions. Parmi ses nombreuses dispositions, on retiendra son article ler créant l'article L. 3136-2 du Code de la santé publique 
aux termes duquel « L'article 121-3 du Code pénal est applicable en tenant compte des compétences, $d u$ pouvoir et des moyens dont disposait l'auteur des faits dans la situation de crise ayant justifié l'état d'urgence sanitaire, ainsi que de la nature de ses missions ou de ses fonctions, notamment en tant qu'autorité locale ou employeur ». Les sénateurs requérants considéraient que cette disposition méconnaissait le principe d'égalité devant la loi dès lors qu'elle pouvait avoir " pour effet d'exonérer certains « décideurs » de toute responsabilité pénale ». Le Conseil écarte l'argument après avoir rappelé le contenu des alinéas 3 et 4 de l'article 121-3 du Code pénal que le Code de la santé publique se propose d'appliquer. Selon lui, le nouveau texte ne s'écarte pas des règles posées par le Code pénal de sorte que, d'une part, il n'est pas différent de l'article 121-3 du Code pénal et que, d'autre part, il a vocation à s'appliquer de la même façon à tous ceux qui, lors de la crise sanitaire, auraient pris des décisions constitutives d'une faute non intentionnelle. Il est vrai que l'article L. 3136-2 ne modifie aucunement la règle posée par l'article 121-3 puisqu'il se borne à en prévoir l'application. De même, il ne fait que préciser les conditions d'application du texte de droit commun : il faut tenir compte des compétences, du pouvoir et des moyens dont disposait l'auteur des aits dans la situation de crise ayant justifié l'état d'urgence sanitaire et apprécier la nature de ses missions ou de ses fonctions « en tant qu'autorité locale ou employeur ». Cette appréciation doit simplement s'effectuer par rérence à la période de l'état d'urgence sanitaire puisque le texte renvoie aux pouvoirs, compétences et moyens dont l'agent disposait lors de la crise sanitaire, ce qui serait également le cas par application du seul article 121-3 du Code pénal.

En conclusion, cette disposition ne sert à rien et l'on peut s'étonner de l'initiative d'un législateur qui avait pourtant autre chose à faire dans le contexte de crise que la France connaissait à l'époque (P. Conte, Un droit en trompe-l'œil : Dr. pén. 2020, étude 19).

\section{Cons. const., déc. $n^{\circ}$ 2020-801 DC du 18 juin 2020, Loi visant à lutter contre les contenus haineux sur internet. Retrait de contenus à caractère terroriste ou pédopornographique, de propos haineux ou à caractère sexuel. Non-conformité de l'article 1er \$1 et 2, par V. PELTIER}

Avant d'être promulguée, la loi Avia n 2020-766 du 24 juin 2020 visant à lutter contre les contenus haineux sur internet a été déférée devant le Conseil constitutionnel par les sénateurs qui lui faisaient un certain nombre de griefs. Au titre des dispositions pénales litigieuses, on retiendra l'article $1^{\text {er }}$ de la loi, dans ses paragraphes 1 et 2 . Tout d'abord, le paragraphe 1 modifiait l'article 6-4 de la loi du 21 juin 2004 qui autorise l'autorité administrative à demander aux hébergeurs ou aux éditeurs d'un service de communication en ligne de retirer certains contenus à caractère terroriste ou pédopornographique et qui, en l'absence de retrait dans un délai de vingt-quatre heures, lui permet de notifier la liste des adresses des contenus incriminés aux fournisseurs d'accès à internet qui doivent alors sans délai en empêcher l'accès. Le nouveau texte réduisait le délai de vingt-quatre à une heure en punissant le manquement à cette obligation de retrait d'un an d'emprisonnement et de 250000 euros d'amende. Ensuite, le paragraphe 2 créait un article 6-2 dans la loi du 21 juin 2004 imposant à certains opérateurs de plateforme en ligne, sous peine de sanction pénale, de retirer ou de rendre inaccessibles dans un délai de vingt-quatre heures des contenus illicites en raison de leur caractère haineux ou sexuel.

Dans les deux cas, le Conseil va conclure à l'absence de conformité, car l'atteinte portée à l'exercice de la liberté d'expression et de communication n'est ni nécessaire, ni adaptée et proportionnée. Autrement dit, la balance des intérêts entre liberté 
de communication - impliquant la liberté d'accéder et d'utiliser des services de communication en ligne - et l'atteinte à l'ordre public et aux droits des tiers a, dans ces hypothèses, nettement penché en faveur de la suprématie de la liberté d'expression.

Il est vrai qu'aux termes du $\S 1$, le Conseil ne pouvait naturellement se satisfaire du laps de temps laissé aux hébergeurs ou aux éditeurs pour retirer les contenus litigieux dans la mesure où la procédure semblait teintée d'arbitraire. En effet, d'abord, c'est l'autorité administrative qui décidait de l'illicéité du contenu à retirer, ensuite, le recours contre une telle décision n'étant pas suspensif, l'hébergeur se voyait obligé de procéder au retrait sans qu'un juge ait pu se prononcer et, enfin, s'il ne le aisait pas dans ce délai extrêmement contraint d'une heure, il encourait de fortes peines, pas forcément pertinentes au demeurant : un an d'emprisonnement et 250000 euros d'amende. Le Conseil abroge par conséquent le $\S$ $1^{\text {er }}$ de l'article 1 sans que sa décision ait à faire l'objet d'autre commentaire.

C'est le même type de griefs qui vont avoir raison du $\S 2$ du texte tant le législateur avait créé une procédure inique : l'opérateur devait retirer tous les contenus manifestement illicites qui lui auraient été signalés, sans intervention d'un juge, en les qualifiant lui-même pénalement sans se contenter de reprendre le motif allégué pour le retrait (avec toutes les difficultés d'appréciation de l'illicéité manifeste que connaissent les juristes pénalistes), le tout dans un délai de 24 heures sous peine de sanctions pénales... En l'absence de cause d'exo-nération, cette disposition aurait donc conduit les opérateurs à retirer tous les contenus sans distinction, ce qui porte à la liberté d'expression une atteinte dont la disproportion est elle aussi manifeste, d'où la censure logique du Conseil constitutionnel. En d'autres termes, dans un État de droit, la poursuite d'un objectif, si louable soit-il (lutte contre le terrorisme, la pédopornographie et les contenus haineux) ne peut s'effectuer à n'importe quel prix. Pas encore... Là aussi, la solution n'ap-pelle aucun commentaire supplémentaire tant elle est logique, mais invite à s'interroger sur l'incurie législative : personne n'avait donc averti la personne chargée de porter la loi sur les contenus haineux de l'inconstitutionnalité manifeste des dispositions qu'elle contenait?

La question est d'autant plus préoccupante que l'on notera que le Conseil constitutionnel censure également un certain nombre de dispositions contenues dans le projet de loi, non pas par non-conformité substantielle à la Constitution, mais du fait des modalités de leur adoption. En effet, un certain nombre de dispositions n'ont aucun rapport, direct ou indirect, avec l'objet de la loi et sont donc déclarées non conformes à la Constitution, car adoptées selon une procédure qui lui est contraire (toutes les dispositions avaient été introduites en première lecture de la loi sans avoir aucun lien avec son objet). Il s'agit des articles 11 (modifiant les articles 138 du Code de procédure pénale et 132-45 du Code pénal afin de compléter la liste des obligations susceptibles d'être prononcées dans le cadre d'un contrôle judiciaire ou d'un sursis probatoire, en y ajoutant l'interdiction d'adresser des messages, notamment électroniques, à la victime) et $122^{\circ}$ et $3^{\circ}$ (modifiant les dispositions des articles 510 et 512 du Code de procédure pénale relatives à l'appel des décisions rendues à juge unique en matière correctionnelle) de la loi. 


\section{Procédure pénale}

Cons. const., déc. n 2019-822 QPC 24 janvier 2020, - M. Hassan $S$. (Absence d'obligation légale d'aviser le tuteur ou le curateur d'un majeur protégé entendu librement). Non-lieu à statuer, par Évelyne BoNIS

La décision $n^{\circ}$ 2019-822 QPC aborde une question désormais bien connue dans la jurisprudence du Conseil constitutionnel pour avoir été déjà examinée, en des termes semblables, lors d'une précédente décision du 14 septembre 2018 (Cons. const., déc. $\mathrm{n}^{\circ}$ 2018-730 QPC du 14 sept. 2018, M. Mehdi K., v. nos obs. cette revue, ${ }^{\circ} 1$ et 2, oct. 2018 avril 2019, p. 321-328) : celle de l'information du tuteur ou du curateur d'un majeur protégé de la procédure pénale dont celui-ci fait l'objet. L'intérêt principal de la décision du 24 janvier 2020 n'est pas tant de se prononcer sur la conformité de l'article 706-113 du Code de procédure pénale aux droits et libertés fondamentaux, question pour laquelle le Conseil constitutionnel a déjà procédé à une déclaration de non-conformité, mais de rappeler les règles régissant l'autorité attachée à ses décisions.

En l'occurrence, l'argumentation du requérant à l'appui du grief d'inconstitutionnalité de l'article 706-113 du Code tel qu'il résultait de la loi du 25 février 2008 consistait à soutenir que ces dispositions méconnaissaient les droits de la défense en cas d'audition libre d'un majeur protégé, car elles n'imposent pas à l'officier de police judiciaire d'aviser son curateur ou son tuteur, non plus que le juge des tutelles, de la mesure d'audition dont la personne fait l'objet. La personne protégée ne disposant pas toujours du discernement nécessaire à l'exercice de ses droits, l'absence de cette garantie serait susceptible de l'amener à opérer des choix contraires à ses intérêts.

Elle est à cet égard quasi identique à celle présentée à l'occasion de la précédente QPC examinée le 14 septembre 2018 à cette différence près qu'elle vise l'audition libre et non, comme auparavant, la garde à vue. Lors de la question prioritaire soumise en 2018 au Conseil, le requérant soutenait en effet que l'article 706-113, pris également dans sa rédaction résultant de la loi du 25 février 2018 « méconnaîtraient les droits de la défense au motif que, en cas de placement en garde à vue d'un majeur protégé, elles n'imposent pas à l'officier de police judiciaire d'aviser son curateur ou son tuteur, ainsi que le juge des tutelles. La personne protégée ne disposant pas toujours du discernement nécessaire à l'exercice de ses droits, l'absence de cette garantie ne saurait être suppléée, lors de son placement en garde à vue, par la seule notification de son droit de faire prévenir son curateur ou son tuteur ».

Si garde à vue et audition libre sont deux mesures pouvant être ordonnées, au stade policier, à l'endroit d'une personne à l'encontre de laquelle il existe des raisons plausibles de soupçonner qu'elle a commis ou tenté de commettre une infraction (C. pr. pén., art.62), elles sont bien différentes, la garde à vue étant une mesure bien plus attentatoire aux droits et libertés puisqu'elle est contraignante (C. pr. pén., art. 62-2), à l'inverse de l'audition libre qui suppose le consentement de la personne à être entendue (C. pr. pén., art. 61-1). La question soulevée par le requérant pouvait donc se comprendre et cela d'autant plus que dans sa décision de 2018, le Conseil avait raisonné par rapport à la capacité de la personne à exercer ses droitls, faute de discernement suffisant ou de possibilité d'exprimer sa volonté en raison de l'altération de ses facultés mentales ou corporelles. Il avait ainsi fait état du fait que le gardé à vue est susceptible d'opérer des choix contraires à ses intérêts, au regard notamment de l'exercice de son droit de s'entretenir avec un avocat et d'être assisté par lui au cours de ses auditions et confrontations. Or, si l'infraction est un crime ou un délit puni d'une peine 
d'emprisonnement, la personne entendue librement dispose également du droit d'être assistée au cours de son audition ou de sa confrontation, par un avocat choisi par elle ou, à sa demande, désigné d'office par le bâtonnier de l'ordre des avocats (C. pr. pén. art. $61-1,5^{\circ}$ ). Pour elle aussi, il y a donc lieu de craindre un choix non éclairé si ses facultés mentales sont altérées.

Le Conseil constitutionnel ne dit pas le contraire dans la décision du 24 janvier 2020 au terme d'un raisonnement qui ne consiste pas à vérifier la conformité des dispositions applicables à l'audition libre à la Constitution, mais à se fonder sur l'autorité attachée à ses décisions. Il rappelle ainsi que cette autorité des décisions s'attache non seulement à leur dispositif, mais aussi aux motifs qui en sont le soutien nécessaire et en constituent le fondement même. En l'absence de changement des circonstances, cette autorité fait obstacle à ce qu'il soit saisi d'une question prioritaire de constitutionnalité relative à une disposition déclarée contraire à la Constitution. Or, la divergence dans l'argumentation du requérant ne traduit pas un changement de circonstances justifiant qu'il soit de nouveau saisi afin d'examiner la conormité à la Constitution de ces dispositions, dans la même rédaction du texte. En outre, le fait que dans la décision de septembre 2018, le Conseil ait décidé que la déclaration d'inconstitutionnalité ne prendrait effet, sous certaines conditions, qu'au $1^{\text {er }}$ octobre 2019 ne change rien à l'autorité attachée à la décision. Le Conseil conclut ainsi n'y avoir pas lieu de statuer sur la question prioritaire de constitutionnalité portant sur le premier alinéa de l'article 706-113 du Code de procédure pénale, dans sa rédaction résultant de la loi ${ }^{\circ}$ 2008-174 du 25 février 2008 relative à la rétention de sûreté et à la déclaration d'irresponsabilité pénale pour cause de trouble mental.

La situation, et la solution sans doute aussi, aurait été très différente si le recours n'avait pas eu pour objectif de s'assurer de la conformité de l'article 706-113 dans sa rédaction issue de la loi de 2008 à la Constitution, mais dans la nouvelle rédaction des dispositions relatives à la poursuite, à l'instruction et au jugement des infractions commises par des majeurs protégés issue de la loi $\mathrm{n}^{\circ}$ 2019222 du 23 mars 2019. En effet, on rappellera qu'à la suite de la décision de non-conformité du 14 septembre 2018, et comme l'y invitait le Conseil constitutionnel, le législateur est intervenu pour réformer le Code de procédure pénale et ajouter un article 706-112-1 spécifique à la question de l'information du curateur ou du tuteur d'un majeur protégé faisant l'objet tant d'une mesure de garde à vue (pour répondre ainsi à la décision de 2018) ainsi qu'un article 706-112-2 spécifique à l'audition libre (anticipant ainsi sur la décision de 2020). Aux termes de ce dernier article et à compter du $1^{\text {er }}$ juin 2019, «Lorsque les éléments recueillis au cours d'une procédure concernant un crime ou un délit puni d'une peine d'emprisonnement font apparaître qu'une personne devant être entendue librement en application de l'article 61-1 fait l'objet d'une mesure de protection juridique, l'officier ou l'agent de police judiciaire en avise par tout moyen le curateur ou le tuteur, qui peut désigner un avocat ou demander qu'un avocat soit désigné par le bâtonnier pour assister la personne lors de son audition. Si le tuteur ou le curateur n'a pu être avisé et si la personne entendue n'a pas été assistée par un avocat, les déclarations de cette personne ne peuvent servir de seul fondement à sa condamnation $»$. Cette disposition - qui n'était pas en vigueur à la date de la procédure diligentée contre le requérant puisque, selon l'arrêt de renvoi au Conseil rendu par la chambre criminelle de la Cour de cassation le 29 octobre 2019 (Cass. crim., 29 oct. 2019, pourvoi n`19-90030), la question prioritaire de constitutionnalité avait été transmise par arrêt de la Cour d'appel d'Aixen-Provence, en date du 25 juillet 2019, dans une procédure suivie du chef de vol en récidive contre le requérant - permet désormais de s'assurer de la préservation des droits du majeur sous un régime civil de protection faisant l'objet d'une audition libre. 
Cons. const., déc. n²019-828/829 QPC du 28 février 2020, $M$. Raphaël S. et autre (Déposition sans prestation de serment pour le conjoint de l'accusé) - Non-conformité de l'article $335,5^{\circ}$ du Code de procédure pénale, par Évelyne BonIS

Selon l'article 331 du Code de procédure pénale relatif au déroulement de la procédure devant les cours d'assises, avant de commencer leur déposition, les témoins prêtent le serment « de parler sans haine et sans crainte, de dire toute la vérité, rien que la vérité ». Ce n'est qu'une fois cette pres-tation de serment opérée, qu'ils sont admis à dépo-ser oralement devant la Cour. Toutefois, l'article 335 du même Code dresse une liste de personnes dont le témoignage peut être reçu sans qu'elles aient au préalable à prêter serment. Il s'agit : " $1^{\circ}$ Du père, de la mère ou de tout autre ascendant de l'accusé, ou de l'un des accusés présents et sou-mis au même débat ; $2^{\circ} \mathrm{Du}$ fils, de la fille, ou de tout autre descendant ; $3^{\circ}$ Des frères et sœurs ; $4^{\circ}$ Des alliés aux mêmes degrés ; $5^{\circ} \mathrm{Du}$ mari ou de la femme; cette prohibition subsiste même après le divorce ; $6^{\circ}$ De la partie civile; $7^{\circ}$ Des enfants au-dessous de l'âge de seize ans ; $8^{\circ}$ De toute personne qui a été accusée, prévenue ou condam-née soit pour le crime dont est saisie la cour d'as-sises en qualité de coauteur ou de complice, soit pour un crime ou un délit connexe ou formant un ensemble indivisible avec le crime dont est saisie la cour d'assises ». Cette dispense de prêter ser-ment est lourde de sens pour les témoins, car, non seulement elle simplifie leur situation en allégeant le processus de déposition, mais surtout elle les fait échapper aux risques pénaux attachés à une déclaration faite sous serment puisqu'ils ne pour-ront pas être poursuivis pour les infractions de refus de prêter serment (C. pr. pén., art. 326) ou pour faux témoignage sous serment (C. pén., art. 434-13).
C'est à propos de la rédaction du $5^{\circ}$ de cet article 335 que le Conseil a dû se prononcer le 28 février 2020 puisqu'il était alors saisi de deux questions prioritaires de constitutionnalité jointes et transmises par la chambre criminelle de la Cour de cassation le 11 décembre 2019 (Cass. crim., 11 déc. 2019, pourvois $n^{\circ} 19-80361$ et $n^{\circ} 18-84049$ ). Rédigés à l'identique, ces deux arrêts de renvoi mettaient en avant l'objectivité et la sincérité du témoignage qui peuvent être affectées par le fait que le témoin et l'accusé ont des conditions de vie commune et cela quel qu'en soit le statut juridique, mariage certes comme en dispose l'article 335, mais aussi pacte civil de solidarité ou concubinage. Devant le Conseil, les requérants invoquaient ainsi la différence de traitement entre, d'une part, les époux et, d'autre part, les concubins ou les partenaires liés par un pacte civil de solidarité, qui sont, eux, soumis à une obligation de prêter serment. Il en résultait selon eux une violation des principes d'égalité devant la loi et devant la justice. En outre, pour l'un des deux requérants, en privant les concubins de la possibilité de témoigner sans prêter serment, à titre de simples renseignements et sans donc encourir le risque d'être poursuivi pour faux témoignage, ces dispositions porteraient atteinte aux droits de la défense. Ce second moyen n'a pas été examiné par le Conseil, le premier ayant suffi à déclarer l'article $335,5^{\circ}$ non conforme à la Constitution.

À l'heure où la plupart des dispositions du Code de procédure pénale ont vu leur écriture modifiée dans le but de tenir compte de la diversité des formes d'union juridiquement reconnues (v. par ex., en ce sens, C. pr. pén., art. 41-2, 41-1, 41-3-1 à propos des attributions du procureur de la République ou encore 138 à propos du contrôle judiciaire, lesquels visent systématiquement les infractions commises soit contre son conjoint, son concubin ou son partenaire lié par un pacte civil de solidarité, soit contre ses enfants ou ceux de son conjoint, concubin ou partenaire), l'article 335 peut sembler assez anachronique en ce qu'il ne vise que la forme du mariage. Pourtant, si le Conseil constitutionnel conclut bien à sa nonconformité à la Constitution dans cette décision 
du 28 février 2020, il convient de remarquer qu'il le fait au terme d'un raisonnement plutôt scrupuleux à partir de sa définition classique du principe d'égalité devant la loi tel qu'il résulte de l'article 6 de la Déclaration des droits de l'homme et du citoyen. Il rappelle ainsi que le principe d'égalité devant la loi ne s'oppose ni à ce que le législateur règle de aon différente des situations différentes ni à ce qu'il déroge à l'égalité pour des raisons d'intérêt général, pourvu que, dans l'un et l'autre cas, la différence de traitement qui en résulte soit en rapport direct avec l'objet de la loi qui l'établit. Son raisonnement s'opère ainsi en deux temps.

Dans un premier temps, il recherche s'il existe une différence de situations entre les personnes selon la forme juridique de leur union ce qui le conduit à remarquer que l'intensité des droits et obligations qui s'imposent aux membres du couple diffère selon qu'ils choisissent l'une ou l'autre de ces unions. D'une manière générale, il n'y a donc pas une situation identique selon les formes de l'union. Néanmoins, au regard de la question de la dispense de serment, il en appelle, comme la Cour de cassation avant lui, à la raison d'être de la dispense créée pour les époux à savoir à la dimension morale. Il indique ainsi qu'en instaurant une telle dispense, "le législateur a entendu préserver le conjoint appelé à témoigner du dilemme moral auquel il serait exposé s'il devait choisir entre mentir ou se taire, sous peine de poursuites, et dire la vérité, pour ou contre la cause de l'accusé » (motif $\mathrm{n}^{\circ} 10$ ) pour conclure que les personnes engagées dans des formes d'union autres que le mariage ne sont pas moins exposées que les conjoints au dilemme moral dont le législateur a entendu préserver ces derniers lorsqu'ils sont appelés à témoigner au procès de leur conjoint accusé. Dès lors, il faut en déduire que du point de vue de la dispense de peine, les diverses personnes ne sont pas dans une situation différente qui justifierait une diférence de traitement. Elles devraient donc faire l'objet d'un traitement identique, sauf à ce que des raisons d'intérêt général justifient qu'il soit dérogé à cette égalité.
Dans un second temps, le Conseil examine ainsi l'existence de potentielles raisons d'intérêt général ce qui le conduit à s'intéresser à l'efficacité de la procédure devant la cour d'assises, plus spécialement à "l'intérêt qui s'attache à ce que la cour d'assises puisse facilement s'assurer de l'existence ou non du lien du témoin avec l'accusé ». Cet intérêt pour le moins original et inédit dans la jurisprudence du Conseil est identifié en raison des enjeux de cette vérification puisqu'une déposition effectuée sans prêter serment alors que le témoin était tenu de le faire est susceptible de vicier la procédure. Cette nécessité d'une vérification aisée par la cour d'assises est présente dans la jurisprudence de la Cour de cassation qui fait de la preuve de ce lien un préalable nécessaire à son contrôle du respect de la procédure. Elle exige ainsi que le procès-verbal des débats précise le lien de parenté ou d'alliance qui unit le témoin et l'accusé (V. en ce sens Cass. crim., 21 juin 1995 : pourvoi n $94-85194$ : Bull. crim. n²28, déc. à laquelle se réfère le Cons. dans ses visas. V. également auparavant Cass. crim., 22 oct. 1969 : n6990917 : Bull. crim. n²61). À défaut d'être indiquée dans le procès-verbal des débats, la preuve de ce lien peut être recherchée dans les pièces de la procédure et notamment dans les énonciations de l'arrêt de renvoi (Cass. crim., 18 déc. 1968, ${ }^{\circ}$ 68-91.105 : Bull. Crim. n॰348 ; Cass. crim., 25 juin 1980, n 79-93.314 : Bull. crim., $n^{\circ}$ 207). La preuve de ce lien est aisée en cas de mariage compte tenu de la publicité dont il fait l'objet. Il l'est cela dit tout autant, indique le Conseil, en cas de pacte civil de solidarité ou de concubinage. Le Conseil relève ainsi que le pacte civil de solidarité fait l'objet d'un enregistrement en mairie. La situation est plus délicate pour le concubinage qui, selon l'article 515-8 du Code civil, est défini comme une union de fait, caractérisée par une vie commune présentant un caractère de stabilité et de continuité, entre deux personnes qui vivent en couple. Le Conseil considère toutefois que « compte tenu de ces critères de stabilité et de continuité, la cour d'assises est en mesure, au regard notamment des éléments recueillis lors de l'instruction, de s'assurer de l'existence d'une vie commune constitutive d'un concubinage ». Aussi, et nonobstant 
la différence des modalités de preuve du lien, le Conseil conclut que l'intérêt qui s'attache à faciliter la connaissance par la juridiction des liens unissant l'accusé et le témoin ne saurait, à lui seul, justifier la différence de traitement établie par les dispositions contestées entre le mariage, le concubinage et le pacte civil de solidarité. Il conclut ainsi à la non-conformité de l'article $335,5^{\circ}$ du Code de procédure pénale à la Constitution. Toutefois, et comme l'y autorise l'article 62 de la Constitution, il décide de reporter au 31 décembre 2020 la date de l'abrogation des dispositions contestées, car l'abrogation immédiate des dispositions déclarées priverait les époux d'une garantie. Ils perdraient en effet le bénéfice de la dispense de prestation de serment ce qui serait de nature à entraîner des conséquences manifestement excessives.

En attendant une intervention du législateur qui le conduira sans doute à élargir la liste des bénéficiaires de la dispense aux concubins et aux partenaires liés par un pacte civil de solidarité, cette décision du 28 février 2020 comme d'ailleurs avant elles, les deux décisions de renvoi de la Cour de cassation, fera date, car elles marquent une évolution assez nette dans la jurisprudence. Jusque-là, la Cour de cassation avait à deux reprises, refusé de transmettre au Conseil des questions, il est vrai différentes, mais portant sur la conformité de l'article 335 (Cass. crim., 3 oct. 2018, nº18-81.951 à propos de la différence prétendue de traitement entre la famille de l'accusé et la famille de la partie civile - Cass. crim., 20 juin 2018, n 17-87.327, à propos de la situation de personnes placées sous un régime civil de protection). Elle marque surtout une réelle volonté d'ouverture de la part de la Cour de cassation qui jusqu'alors, et à juste titre, s'en tenait à la stricte lettre de l'article 335 et donc au caractère limitatif de la liste. Cette liste constituant une exception par rapport au principe qui veut que le témoin prête serment, elle était de droit étroit ce qui avait conduit la Cour de cassation à refuser, de façon systématique, de l'élargir (v. par ex. pour un refus d'extension à l'épouse du frère du mari de l'accusée, laquelle n'est pas un allié au sens de l'article $335,4^{\circ}$ : Cass. crim., 21 déc.
1972 : Bull. crim. n 399 ; 28 avril 1978 : Bull. crim. $n^{\circ} 131 ; 14$ oct. 1992, n91-86.985 : Bull. crim. $\left.n^{\circ} 323\right)$.

Cette intervention législative pourrait aussi être l'occasion d'un toilettage plus ample du Code de procédure pénale, car, au-delà de la procédure devant la cour d'assises, l'article 448 du Code relatif au témoignage devant le tribunal correctionnel dispose également que les dépositions se font sans prestation de serment pour une liste limitative de personnes dont, " $5^{\circ} \mathrm{Du}$ mari et de la femme ». La Cour de cassation opère là aussi une interprétation stricte de cet alinéa ce qui a pu la conduire à exclure que l'on entende sans prestation de serment, la concubine du prévenu dans un arrêt rendu le 5 janvier 2017 (Cass. crim., 5 janv. 2017 : pourvoi $\left.\mathrm{n}^{\circ} 15-85.921\right)$. Il semble bien que les motifs avancés par le Conseil à l'appui de la déclaration de non-conformité de l'article $335,5^{\circ}$ à la Constitution puissent être reproduits à l'endroit de cet article $448,5^{\circ}$ du Code. Le législateur serait ainsi bien inspiré que de réécrire ces deux alinéas.

\section{Cons. const., déc. n' 2020 -} 836 QPC du 30 avril 2020, M. Maxime O. (Utilisation de la
visioconférence sans accord du détenu dans le cadre d'audiences relatives au contentieux de la détention provisoire) Non-conformité de l'article 706-71, $4^{\circ}$, par Évelyne BoNIS

Par cette décision rendue le 30 avril 2020, le Conseil constitutionnel se prononce une nouvelle fois sur la question de l'utilisation de la visioconférence sans accord du détenu dans le cadre d'audiences relatives au contentieux de la détention provisoire. Il a en effet déjà eu l'occasion de statuer au sujet de l'article 706-71 du Code de 
procédure pénale dans une précédente décision rendue le 20 septembre 2019, décision à l'occasion de laquelle il avait validé le recours à la visioconférence imposée, dans son principe, pour les audiences relatives aux demandes de mise en liberté, mais abrogé l'alinéa 3 de l'article 706-71, en raison des spécificités de la procédure criminelle (Cons. const., déc. n²019-802 QPC du 20 sept. 2019 , M. Abdelnour B., AJ pénal 2019, n¹2, p. 600 , note J. B. Perrier). Il observait à cette occasion qu'en matière criminelle, la première prolongation de la détention provisoire peut n'intervenir qu'à l'issue d'une durée d'une année de telle sorte qu'en imposant le recours à la visioconférence lors des audiences relatives aux demandes de mise en liberté, « une personne placée en détention provisoire pourrait se voir privée, pendant une année entière, de la possibilité de comparaître physiquement devant le juge appelé à statuer sur la détention provisoire ». Une telle durée a ainsi été vue comme excessive par le Conseil, eu égard à l'importance de la garantie qui s'attache à la présentation physique de l'intéressé devant la juridiction compétente pour connaître de la détention provisoire.

Dans la décision du 30 avril 2020, si de nouveau l'article 706-71 est soumis à l'examen du Conseil, c'est dans une nouvelle rédaction, celle résultant de la loi du 23 mars 2019 de telle sorte que le Conseil ne pouvait dire n'y avoir lieu à statuer en raison de l'autorité attachée à sa précédente décision. Il n'en demeure pas moins que le Conseil s'est considérablement facilité la tâche en statuant par renvoi à sa précédente décision et spécialement aux motifs des paragraphes 7 à 13 . Cette pratique de la motivation par simple et exclusif renvoi à une précédente décision est suffisamment rare dans la pratique du Conseil pour être relevée. Il faut dire néanmoins que si l'on met de côté la forme, la décision est, sur le fond, sans surprise, le législateur ayant fait preuve d'une évidente légèreté à la rédaction du nouvel article 706-71. En effet, il s'est, en quelque sorte, contenté de déplacer de l'alinéa 3 à l'alinéa 4, les dispositions qui avaient été déclarées non conformes à la Constitution dans la décision du 20 septembre 2019. Comme la doctrine avait eu l'occasion de le relever à l'occasion du commentaire de la première décision, une nouvelle QPC, portant cette fois sur l'alinéa 4 de l'article 706-71 était des plus probables et « les mêmes causes devant produire les mêmes effets, le Conseil devrait abroger la disposition litigieuse » (v. op. cit., supra. J. B. Perrier). C'est précisément le sens de la décision du 30 avril 2020 qui déclare l'alinéa 4 de l'article 706-71 non conforme à la Constitution pour les mêmes raisons que celles précédemment visées dans la décision de 2019.

Au-delà de ce qu'elle vient donc confirmer l'importance de la présentation physique de la personne devant un juge, cette solution présente une singularité : elle a une portée a priori plus grande que celle du 20 septembre 2019 dont on rappellera l'apport essentiellement théorique puisque les dispositions déclarées contraires à la Constitution, dans leur rédaction contestée, n'étaient plus en vigueur, au moment où le Conseil les a déclarées non conformes à la Constitution. Il avait en outre précisé que la remise en cause des mesures prises sur le fondement des dispositions déclarées contraires à la Constitution méconnâ̂trait les objectifs de valeur constitutionnelle de sauvegarde de l'ordre public et de recherche des auteurs d'infractions et aurait ainsi des conséquences manifestement excessives de telle sorte que les mesures prises auparavant ne pouvaient être contestées sur le fondement de la décision. À l'inverse, avec la décision du 30 avril 2020, l'abrogation des mots « la chambre de l'instruction » à l'alinéa 4 de l'article 706-71 aurait pu emporter des conséquences concrètes puisque ce texte est en vigueur. Toutefois, le Conseil module de deux manières les effets de sa décision dans le temps. D'une part, il relève qu'une abrogation immédiate des mots « la chambre de l'instruction » aurait pour effet de rendre impossible tout recours à la visioconférence pour les audiences relatives au contentieux de la détention provisoire devant la chambre de l'instruction, ce qui irait au-delà de sa solution laquelle entend limiter à la seule matière criminelle cette impossibilité. Aussi, il décide de reporter au 31 octobre 2020 la date de l'abrogation des dispositions contestées. D'autre part, il estime 
que la remise en cause des mesures prises sur le fondement des dispositions déclarées contraires à la Constitution méconnaîtrait les objectifs de valeur constitutionnelle de sauvegarde de l'ordre public et de recherche des auteurs d'infractions et aurait ainsi des conséquences manifestement excessives. Aussi, il décide que ces mesures ne peuvent être contestées sur le fondement de cette inconstitutionnalité. Une fois encore, la portée de la décision est donc, pour l'heure, limitée.

\section{Droit de la peine}

\section{Cons. const. déc. ${ }^{\circ}$ 2019-827 QPC du 28 févr. 2020, M. Gérard F., (Conditions de recevabilité d'une demande de réhabilitation judiciaire pour les personnes condamnées à la peine de mort). Conformité, par Évelyne BoNIS}

Alors même qu'elle ne suscite qu'un contentieux assez limité devant la Cour de cassation, la réhabilitation judiciaire est l'objet de décisions importantes du Conseil constitutionnel depuis quelques années. Déjà, par une décision rendue le 27 novembre 2015, le Conseil constitutionnel a eu l'occasion de préciser les conditions de la réhabilitation judiciaire d'une peine principale d'interdiction définitive du territoire ranais (Cons. const., déc. no 2015-501 QPC du 27 nov. 2015, M. Anis T. : ; Dr. pénal 2016, comm. 18, obs. V. Peltier). Avec la décision du 28 février 2020, c'est une tout autre hypothèse qui se présentait à lui puisque la peine en question était la peine de mort, une peine que l'on pouvait penser ne plus produire d'effet juridique dans notre droit, pour avoir été abolie par la loi n ${ }^{\circ} 81-908$ du 9 octobre 1981. Pourtant, telle était bien la peine en question dans cette affaire connue sous le nom d'affaire Jacques Fesch qui a conduit le fils d'une personne condamnée à mort par décision de la Cour d'assises de la Seine le 6 avril 1957 et exécutée le $1^{\text {er }}$ octobre 1957 pour un vol à main armée suivi du meurtre d'un gardien de la paix, commis le 25 février 1954 à Paris, à solliciter la réhabilitation judiciaire de son père par le biais d'une requête déposée devant la chambre de l'instruction de la Cour d'appel de Nîmes (C. pr. pén., art. 783, al. 1). Bien que passablement hors délai pour agir, puisque plus de 50 ans se sont écoulés depuis l'expiration de la sanction subie - l'article 785 alinéa $1^{\text {er }}$ prévoit qu'en cas de décès du condamné, les tiers identifiés tels les descendants peuvent agir dans le délai d'une année seulement à dater du décès - , cette requête lui permit de soulever une question prioritaire par laquelle il faisait valoir que les articles 785 et 786 du Code de procédure pénale relatifs aux conditions de dépôt d'une requête en réhabilitation judiciaire aboutissent à priver les proches d'une personne condamnée à la peine de mort de la possibilité de former, en son nom, une telle demande.

Pour bien comprendre son raisonnement, rappelons les termes de ces articles. Le premier de ces deux textes permet d'identifier d'une part, les personnes ayant la qualité pour agir en réhabilitation ainsi que le délai qui leur est ouvert pour le faire. Il dispose que, outre la personne condamnée elle-même, la demande peut être suivie ou formée par le conjoint du défunt ou par ses ascendants ou descendants dans le délai d'une année seulement à compter de la date du décès. Étant le fils du condamné à mort, le requérant avait donc la qualité pour agir en réhabilitation judiciaire. Cependant, agir en 2019 était manifestement bien trop tardif, le délai d'une année étant expiré depuis 1958. C'est précisément ce délai trop court que contestait le requérant qui se prévalait par ailleurs des dispositions de l'article 786 du Code de procédure pénale qui énonce les conditions de recevabilité d'une requête en réhabilitation en posant un délai d'épreuve nécessaire à toute réhabilitation. Plus précisément, selon ce texte, la demande en réhabilitation ne peut être formée qu'après un délai de cinq ans pour les condamnés à une peine criminelle. Ce second délai a pour point de départ le 
jour de l'expiration de la peine criminelle, donc pour une peine de mort, la date de l'exécution. Touteois, est là est l'intérêt essentiel de l'affaire, le requérant entendait souligner l'incohérence de ces textes lorsque la peine criminelle en cause est la peine de mort. L'articulation des deux délais s'avère en effet délicate puisque, par hypothèse même, l'action que les ayants droit du défunt pourraient former dans l'année de l'exécution est vouée à l'échec, la réhabilitation ne pouvant jamais être accordée puisque le délai d'épreuve propre à la réhabilitation judiciaire de 5 ans à compter de la fin de la peine n'est jamais écoulé et même n'a jamais véritablement couru. C'est cette incohérence entre le fait d'ouvrir un délai d'action alors que l'action est vouée à l'échec, faute de pouvoir satisfaire les conditions légales, qui était contestée par le requérant. Selon le moyen, ces dispositions créeraient une différence de traitement entre les personnes selon qu'elles ont été condamnées à la peine de mort - que la peine ait été exécutée ou que ces personnes aient été graciées - ou à une autre peine criminelle, contraire aux principes d'égalité devant la loi et devant la justice d'une part et au principe de proportionnalité des peines d'autre part.

Transmise par les juges du fond à la Cour de cassation, la chambre criminelle jugeait cette question sérieuse pour pas moins de trois raisons (Cass. crim., 11 déc. 2019 : pourvoi n¹9-90031). Tout d'abord, la Cour de cassation raisonne à partir des dispositions légales relatives à la réhabilitation dont elle commence par souligner le caractère général de la réhabilitation, les articles 133-12 du Code pénal et 782 du Code de procédure pénale, permettant que toute personne condamnée par un tribunal français à une peine criminelle, correctionnelle ou contraventionnelle puisse être réhabilitée. Elle souligne alors l'incohérence de notre législation. Elle relève en effet que les articles 785 et 786 du Code de procédure pénale subordonnent la recevabilité de la demande en réhabilitation à des exigences de délais cumulées, qui deviennent incompatibles entre elles lorsque la demande concerne un condamné à mort dont la peine a été exécutée. En effet, l'article 785 prévoit que la demande de réhabilitation doit être présentée du vivant du condamné, ou dans l'année de son décès, alors que l'article 786 exige qu'elle soit présentée après un délai de cinq ans, pour les condamnés à une peine criminelle, ce délai partant, pour les peines autres que l'emprisonnement ou l'amende, prononcées à titre de peine principale, à compter de l'expiration de la sanction subie. Ensuite, la Cour de cassation raisonne par rapport au principe constitutionnel d'égalité, posé par l'article 6 de la Déclaration des droits de l'homme de 1789 pour démontrer que la différence de traitement des condamnés à la peine de mort par rapport aux autres condamnés à une peine criminelle ne lui semble pas justifiée. À cet égard, elle fait valoir deux arguments. D'une part, elle ne voit pas en quoi, de manière évidente, les condamnés à la peine de mort se trouvent, au regard des autres condamnés à des peines criminelles, dans une situation dont la particularité justifie que la réhabilitation leur soit fermée, une telle différence de traitement avec les autres condamnés à une peine criminelle ne paraissant pas en rapport avec l'objet de la loi qui l'a établie. D'autre part, et pour renforcer son propos, elle relève que cette différence de traitement paraît d'autant moins justifiée que l'interdiction constitutionnelle de la peine de mort, résultant de la loi constitutionnelle $\mathrm{n}^{\circ}$ 2007-239 du 23 février 2007, qui a introduit, dans la Constitution, un article 66-1, aux termes duquel nul ne peut être condamné à la peine de mort, peut être de nature à empêcher que les condamnations à mort soient l'objet d'une restriction, conduisant à rendre impossible leur réhabilitation, ouverte à toutes les autres condamnations criminelles.

Cette question prioritaire de constitutionnalité était ainsi transmise au Conseil constitutionnel qui déclara les mots « de cinq ans pour les condamnés à une peine criminelle » figurant à l'article 786, alinéa ${ }^{\text {er }}$, conformes à la Constitution (Cons. const., déc. n 2019-827 QPC du 28 février 2020, M. Gérard F.: Dr. pénal 2020, comm. 92, notre note). Au-delà de la solution, une attention particulière doit être portée à ses motifs. D'une part, le Conseil prend le soin d'expliquer la philosophie 
du mécanisme de la réhabilitation en indiquant qu'il s'agit d'une mesure destinée à favoriser le reclassement du condamné en effaçant toutes les incapacités et déchéances qui résultent d'une condamnation pénale et interdit d'en rappeler l'existence à toute personne (cons. 9) mais aussi et surtout les conditions de la réhabilitation judiciaire qui, contrairement à la réhabilitation de plein droit, ne résulte pas du seul écoulement du temps, mais suppose une appréciation par le juge de la conduite adoptée par le condamné une fois qu'il n'est plus soumis aux rigueurs de la peine prononcée à son encontre (cons. 12). La réhabilitation judiciaire suppose donc, pour être accordée, que, depuis qu'il a subi sa peine, le condamné présente des gages d'amendement. Les condamnés à la peine de mort ne pouvant pas fournir, une fois la peine exécutée, de tels gages, admettre la recevabilité de la demande de réhabilitation judiciaire n'aurait pas de sens puisqu'il serait dans tous les cas, impossible de répondre aux conditions de bien-fondé de la mesure. Cet argument permet ainsi au Conseil de considérer que les condamnés à la peine de mort ne sont pas dans une situation semblable à celle des autres condamnés et que la différence de situation est en rapport avec l'objet de la loi. Il admet ainsi que l'on puisse priver les proches de personnes condamnées à mort de toute possibilité de présenter une demande de réhabilitation judiciaire de leur ayant droit. Toutefois, la mise à l'écart de la procédure de réhabilitation judiciaire n'interdit pas l'instauration d'une autre procédure qui serait spécifique aux condamnés à mort. Le Conseil indique ainsi et d'autre part que le législateur serait fondé à instituer une procédure judiciaire ouverte aux ayants droit d'une personne condamnée à la peine de mort dont la peine a été exécutée tendant au rétablissement de son honneur (cons. $\mathrm{n}^{\circ} 14$ ). Cette procédure $a d$ hoc, dont la finalité serait identique à la procédure de réhabilitation judiciaire - à savoir restaurer dans son honneur la personne - répondrait en revanche à des conditions de bien-fondé différentes puisqu'elle inviterait le juge à apprécier le comportement d'une personne, non plus une fois la peine exécutée pendant le délai d'épreuve propre à la réhabilitation de 5 ans, mais avant la mise à mort soit de la date de la condamnation, voire de la date de commission des faits à la date d'exécution de la peine, ce que la Cour de cassation a toujours refusé fermement, sur le fondement des textes en vigueur, en jugeant : " que si, pour apprécier des gages d'amendement, la chambre de l'instruction peut tenir compte des faits qui ont motivé les condamnations et leur gravité, elle ne peut se fonder uniquement sur ces faits et s'abstenir d'examiner la conduite du condamné pendant le délai prévu par les articles 786 et suivants du code de procédure pénale » (Cass. crim., 11 juill. 2017 : pourvoi $\mathrm{n}^{\circ} 16-86423$, v. auparavant : Cass. crim., 12 février 1963 : Bull. crim. nº 72).

Cette invitation adressée par le Conseil au législateur à créer une telle procédure spécifique pourrait ainsi faire naître de nouveaux espoirs au plan pénal pour le requérant dont le père avait fait montre d'amendement par son engagement religieux durant la procédure pénale. À chacun d'apprécier l'opportunité d'une telle procédure $a d$ $h o c$, voire et au-delà, de l'opportunité de rapprocher le droit et la religion. Il est en effet à noter que depuis le 21 septembre 1987, une instance en béatification a été mise en place par le cardinal Lustiger, archevêque de Paris, afin d'étudier les faits relatifs à la vie et à la conversion de Jacques Fesch (https://fr.zenit.org/2007/10/01/50-ans-dela-mort-de-jacques-fesch-proces-de-beatificationdiocesain-en-cours/). 